\title{
SUPERCONDUCTIVITY IN ANDERSON LATTICE MODEL
}

\author{
A. Romano, C. Noce \\ Unità I.N.F.M. di Salerno -Dipartimento di Fisica Teorica e S.M.S.A. \\ Università di Salerno, 84081 Baronissi (Salerno), Italy \\ AND R. MrCNAS \\ Institute of Physics, A. Mickiewicz University, 60-769 Poznań, Poland
}

\begin{abstract}
We study the superconducting instabilities generated by the inclusion in the Anderson lattice model of a density-density attractive potential between correlated electrons on nearest-neighbouring sites. Using a description of the normal phase based on a perturbative expansion around the atomic limit, we treat the attractive potential in the broken-symmetry Hartree-Fock scheme and analyze which of the possible symmetries of the superconducting order parameter leads to the highest possible transition temperature in the case of a two-dimensional square lattice. For values of the on-site $f$-repulsion large compared to the hopping amplitude, a suppression of any possible superconducting phase occurs, regardless of the symmetry of the order parameter.

PACS numbers: 74.72.-h, 74.70.Tx, 74.25.-q
\end{abstract}

\section{Introduction}

The problem of superconductivity in systems with strong electronic correlations has been intensively studied investigated in the last years, mainly in connection with the unusual properties of the uranium- and cerium-based heavy-fermion superconductors and high- $T_{\mathrm{c}}$ superconducting compounds. Since these materials are characterized by relatively small values of the coherence length, many approaches have focused on the conditions under which correlated electron systems can exhibit nearest-neighbour-pair superconductivity induced by short-range attractive interactions [1].

In this paper we study a generalization of the periodic Anderson model (PAM) which includes a non-retarded nearest-neighbour attraction between correlated $f$-like electrons, which microscopic origin is not investigated here. The compctition between this interaction and the Hubbard on-site repulsion plays a major role in the determination of the superconducting instability, which can develop in given parameter regimes. In particular, we analyze the possible symmetries of the superconducting order parameter, and investigate among them which is the one leading to the highest possible critical temperature. Since the PAM more naturally describes the physical properties of the heavy-fermion systems, the results presented here can be of relevance for characterizing of the superconducting 
phase, which develops at low temperatures in some $U$ and $C e$ compounds. This expectation is also supported by the experimental evidence that Cooper pairs in heavy-fermion superconductors are formed with heavy-mass electrons originating from partially filled $4 f$ and $5 f$ shells.

Former theoretical investigations of heavy-fermion superconductors have used as a starting point similar versions of the generalized PAM studied here [2-3]. A parallel point of view has also been adopted in recent work on the one-band extended Hubbard model [4-8], where the effects of the competition between intrasite repulsive and intersite attractive density-density interactions were studied in the context of theories of high- $T_{c}$ superconductivity.

\section{Model}

The Hamiltonian of a generalized PAM is given by

$$
\begin{aligned}
& H=\left(\epsilon_{f}-\mu\right) \sum_{i, \sigma} f_{i \sigma}^{\dagger} f_{i \sigma}+U \sum_{i} f_{i \uparrow}^{\dagger} f_{i \uparrow} f_{i \downarrow}^{\dagger} f_{i \downarrow}+\left(\epsilon_{c}-\mu\right) \sum_{i, \sigma} c_{i \sigma}^{\dagger} c_{i \sigma} \\
& +V \sum_{i, \sigma}\left(f_{i \sigma}^{\dagger} c_{i \sigma}+c_{i \sigma}^{\dagger} f_{i \sigma}\right)+\sum_{i \neq j, \sigma} t_{i j} c_{i \sigma}^{\dagger} c_{j \sigma}+\frac{1}{2} \sum_{i \neq j, \sigma \sigma^{\prime}} W_{i j} f_{i \sigma}^{\dagger} f_{i \sigma} f_{j \sigma^{\prime}}^{\dagger} f_{j \sigma^{\prime}} .
\end{aligned}
$$

The density-density interaction between $f$-electrons is assumed to be repulsive on the same site $(U>0)$ and attractive on nearest-neighbour sites $\left(W_{i j}<0\right)$. In absence of this latter term, the model reduces to the standard periodic Anderson model. Considering a two-dimensional square lattice with nearest-neighbour hopping, we have $\epsilon(k)=-t \gamma(k)$ and $W(k)=-W \gamma(k)$ with $t, W>0$ and $\gamma(k)=2\left(\cos k_{x}+\cos k_{y}\right)$. With the usual Hartree-Fock factorization, the bare site energy $\epsilon_{f}$ is renormalized by the Hartree term, i.e. $\epsilon_{f} \rightarrow \tilde{\epsilon}_{f}=\epsilon_{f}-W z n_{f}, z$ being the lattice coordination number and $n_{f} \equiv \sum_{\sigma}\left\langle f_{i \sigma}^{\dagger} f_{i \sigma}\right\rangle$ with $\left\langle f_{i \uparrow}^{\dagger} f_{i \uparrow}\right\rangle=\left\langle f_{i \downarrow}^{\dagger} f_{i \downarrow}\right\rangle$.

The formation of a superconducting phase with nearest-neighbour Cooper pairs has been analyzed within the broken-symmetry Hartree-Fock-BCS scheme, while looking for the solutions of the $T_{\mathrm{c}}$ equation for singlet and triplet (equal spin) pairing, respectively. Since the potential $W(k-q)$ appearing in the gap equation can be expressed as a sum of orthogonal terms, which under lattice rotation are $s-, d$ - and $p$-like, that is

$$
W(k-q)=\frac{W}{4}[\gamma(k) \gamma(q)+\eta(k) \eta(q)]+2 W\left(\sin k_{x} \sin q_{x}+\sin k_{y} \sin q_{y}\right)
$$

with $\eta(k)=2\left(\cos k_{x}-\cos k_{y}\right)$, we can assume that the order parameter for the singlet case takes the form $\Delta_{s}(k)=\Delta_{0}+\Delta_{\gamma} \gamma(k)+\Delta_{\eta} \eta(k)$, where the three terms refer to on-site, extended $s$-wave and $d$-wave pairing, respectively. In the triplet case the solution for the gap equation is assumed to have the form $\Delta_{t}(k)=$ $\Delta_{t x} \sin k_{x}+\Delta_{t y} \sin k_{y}$.

The $T_{\mathrm{c}}$ equation for $s$-wave pairing is given by

$$
\left[1+U \phi_{0}\left(T_{\mathrm{c}}\right)\right]\left[4-W \phi_{2}\left(T_{\mathrm{c}}\right)\right]+U W \phi_{1}^{2}\left(T_{\mathrm{c}}\right)=0,
$$

where $\phi_{n}\left(T_{\mathrm{c}}\right)=N^{-1} \sum_{k} \gamma^{n}(k) F\left(k, T_{\mathrm{c}}\right)$ with $F\left(k, T_{\mathrm{c}}\right)=k_{\mathrm{B}} T_{\mathrm{c}} \sum_{\nu} G_{f f}\left(k, \omega_{\nu}\right)$ $\times G_{f f}\left(-k,-\omega_{\nu}\right)$. We have solved it in the general case as well as in the case in which the contribution of the anomalous averages coming from the $U$ term is neglected (what amounts to put $U=0$ in Eq. (3)). 
For $d$-wave pairing $T_{\mathfrak{c}}$ is determined from the equation

$$
\frac{4}{W}=\frac{1}{N} \sum_{k} \eta^{2}(k) F\left(k, T_{\mathrm{c}}\right),
$$

while for the $p$-wave case we have

$$
\frac{1}{2 W}=\frac{1}{N} \sum_{k} \sin ^{2} k_{x} F\left(k, T_{\mathrm{c}}\right) .
$$

The above equations contain the "normal state" Green function $G_{f f}$, not including the anomalous a verages coming from the Hartree-Fock factorization of the intersite attractive potential.

The onset of the superconducting instability is determined by solving self-consistently the $T_{\mathrm{c}}$ equation for each of the possible symmetries of the order parameter, together with the equations fixing the total electron density $n=$ $n_{f}+n_{c}=2\left(k_{\mathrm{B}} T / N\right) \sum_{k, \nu}\left[G_{f f}\left(k, \omega_{\nu}\right)+G_{c c}\left(k, \omega_{\nu}\right)\right]$ and the $f$-electron density $n_{f}$. The normal state Green functions $G_{f f}$ and $G_{c c}$ are evaluated by means of an expansion around the atomic limit [9], which at the lowest order is equivalent to the Hubbard I approximation. Compared with standard mean-field approaches, this choice is expected to lead to a more reliable description of the model in the intermediate and in the large- $U$ regime.

\section{Results and discussion}

The results presented here refer to the case of total electron density close or equal to two electrons per site. In such a case both the $f$-electron $G_{f f}$ and $c$-electron $G_{c c}$ Green functions exhibit four poles which arise from atomic transitions connecting the two-particle ground state with one- and three-particle levels [9] (this holds up to temperatures not so high to give rise to transitions between excited levels). Moreover, we have fixed everywhere $\epsilon_{c}=0$ and $\epsilon_{f}=-0.5$, and all the energies are measured in units of the unperturbed half-bandwidth $D=4 t$.

In the limit of zero on-site Coulomb repulsion the PAM becomes exactly solvable, with an excitation spectrum consisting of two bands separated by a hybridization gap. For realistic values of the hybridization coupling, the superconducting phase showing the highest $T_{c}$, at any density below half filling $(n<2)$ is characterized by the $d$-wave symmetry of the order parameter. No solution for the $T_{\mathrm{c}}$ equations is found for $n \geq 2$, owing to the fact that, for $U=0$ and with the site energy $\epsilon_{f}$ close to the bottom of the conduction band, densities $n>2$ always imply a double $f$-occupation per site, which prevents $f$-electron itinerancy.

A finite value of the on-site repulsion $U$ introduces remarkable changes to this scenario. First of all, when $U$ starts exceeding the unperturbed bandwidth $2 D$, superconductivity tends to be suppressed, regardless of the symmetry of the order parameter. Moreover, the results reported in Fig. 1 show that lower values of $U$ lead to a decrease in $T_{\mathfrak{c}}$, with respect to the case $U=0$, which is more pronounced for $s$ - and $d$ - than for $p$-wave pairing. We have also reported in the same figure $T_{\mathrm{c}}$ for $s$-pairing obtained by putting $U=0$ in Eq. (3) (long-dashed line). For the parameter values chosen, this simplification yields higher values of $T_{c}$, compared to the complete treatment (solid line). This indicates the existence of parameter 


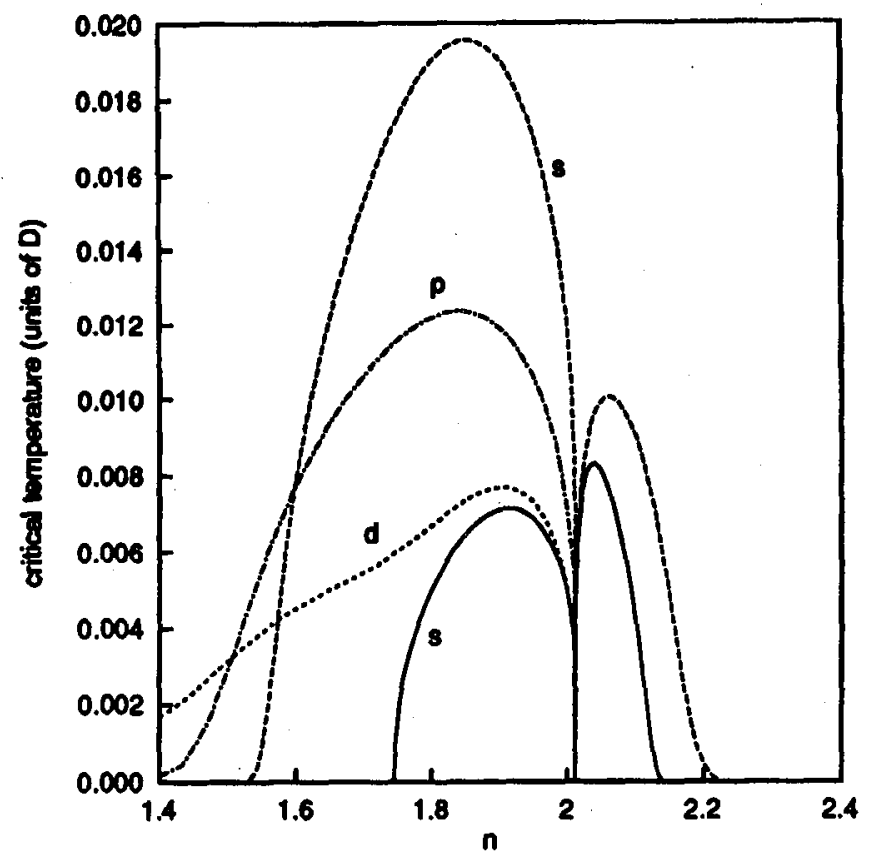

Fig. 1. $T_{\mathrm{c}}$ for anisotropic $s$ - (solid and long-dashed line, see the text), $d$ - (short-dashed line) and $p$-wave pairing (dashed-dotted line) as functions of the total electron density $n$ for $U=1, V=0.2$ and $W=0.15$.

regimes in which the anomalous a verages originated from the $U$ term cannot be neglected. Differently from the case $U=0$, a superconducting instability (with $s$-pairing only) can also occur above the half filling. Indeed, the finite value chosen for $U$ is such that the double $f$-occupation regime is only reached at densities significantly greater than 2 (for the data reported in Fig. 1, at $n \simeq 2.2$ we still have $n_{f} \simeq 1.76$ ). We also note that the relative stability of $s$-, $d$ - and $p$-wave is strongly dependent on the hybridization. Lower values of $V$, for instance, tend to make $s$ - and $d$-pairing more stable than $p$-pairing.

The value of $n$ very close to the half filling, at which $T_{c}$ vanishes for all possible symmetries, corresponds to an insulating state, in which the two lower bands are completely filled and the two upper ones are completely empty, as it can be seen from the corresponding energy spectrum reported in Fig. 2 (the lowest band is indeed a localized level of almost pure $f$-character). The chemical potential lies in the middle of the central gap and its behaviour as a function of $n$ (not reported here for brevity), shows correspondingly a jump, which signals a metal-insulator transition.

The effect introduced by changes of the other parameters of the model (1), competition between superconductivity and phase separation, as well as a more complete characterization of the superconducting phase at half filling will be presented in an extended version of this paper. 


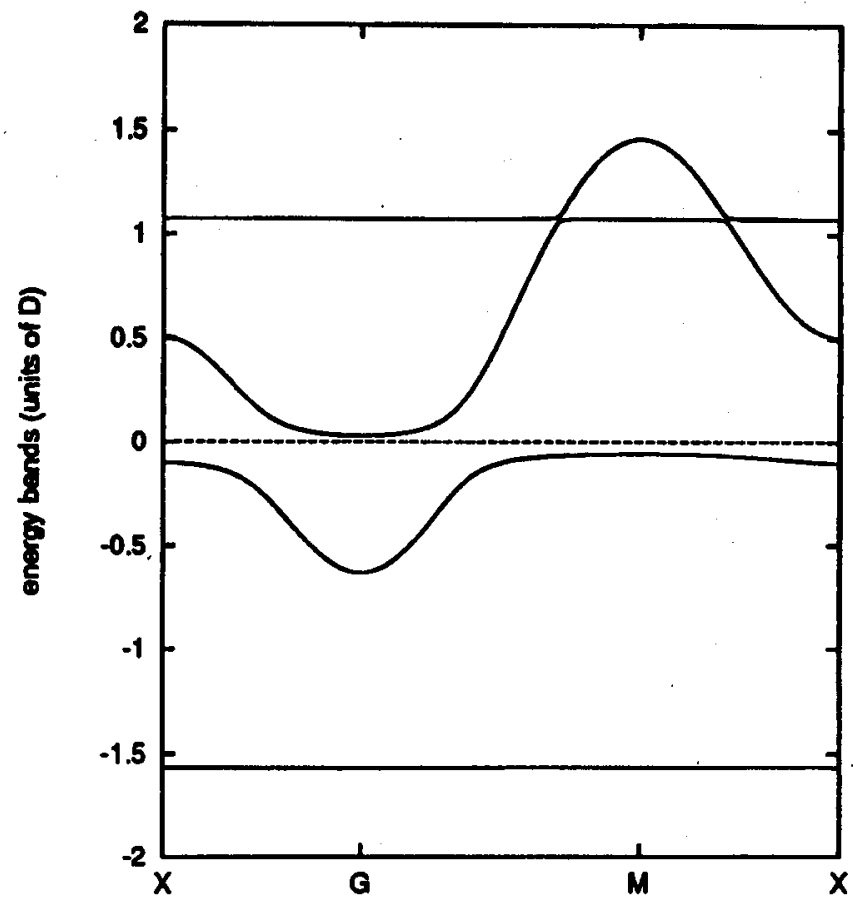

Fig. 2. Band structure in the insulating state close to half filling at which the critical temperatures reported in Fig. 1 vanish (energies are measured with respect to the chemical potential).

\section{Acknowledgments}

This paper has a partial financial support from the Committee for Scientific Research (project numbers 2 P03 B 05709 and 2 P302 038 07).

\section{References}

[1] R. Micnas, J. Ranninger, S. Robaszkiewicz, Rev. Mod. Phys. 62, 113 (1990).

[2] K. Miyake, T. Matsuura, H. Jichu, Prog. Theor. Phys. 72, 652 (1984); K. Miyake, T. Matsuura, H. Jichu, Y. Nagaoka, Prog. Theor. Phys. 72, 1063 (1984).

[3] F.J. Ohkawa, H. Fukuyama, J. Phys. Soc. Jpn. 53, 4344 (1984).

[4] R. Micnas, J. Ranninger, S. Robaszkiewicz, S. Tabor, Phys. Rev. B 37, 9410 (1988);

R. Micnas, J. Ranninger, S. Robaszkiewicz, Phys. Rev. B 39, 11653 (1989).

[5] C. Bastide, C. Lacroix, A. da Rosa Simões, Physica C 159, 347 (1989).

[6] C.A. Balseiro, M.E. Foglio, Phys. Rev. B 40, 9288 (1989).

[7] D.K. Ray, J. Konior, A.M. Oleś, A.N. Das, Phys. Rev. B 43, 5606 (1991).

[8] E. Dagotto, J. Riera, Y.C. Chen, A. Moreo, A. Nazarenko, F. Alcaraz, F. Ortolani, Phys. Rev. B 49, 3548 (1994); S. Haas, E. Dagotto, A. Nazarenko, J. Riera, Phys. Rev. B 51, 5989 (1995).

[9] M. Marinaro, C. Noce, A. Romano, J. Phys., Condens. Matter 3, 3719 (1991). 